\title{
THE SOLUTION OF DUAL SERIES AND DUAL INTEGRAL EQUATIONS
}

\author{
by W. E. WILLIAMS
}

(Received 15 January, 1963)

1. Introduction. There exist several different approaches to the problem of solving dual integral equations involving Bessel Functions $[1,2,3,4,5,6,7]$, and Erdélyi and Sneddon in a recent paper [8] have shown that the introduction of certain operators occurring in the theory of fractional integration enables the relationships between the various methods to be clearly demonstrated. For dual integral equations other than those involving Bessel Functions the operators introduced by Erdélyi and Sneddon are not always the appropriate ones to use and it seems to be of interest to consider this more general type of situation.

The crucial factor in the problem of solving dual series and dual integral equations seems to be the fact that they can be reduced to the solution of an integral equation which can itself then be reduced to the successive solution of two simpler integral equations. This approach seems to have been first used by Copson [9] in obtaining the solution of the electrostatic problem for the circular disk. The crucial factor is thus the existence of a linear operator which can be factorised into the product of two simpler ones. The fractional integral operators introduced by Erdélyi and Sneddon are particular examples of these simpler operators and it is of interest to note that these fractional integral operators can also be applied to the solution of dual series equations involving Jacobi Polynomials. Particular examples of this type of dual series equations have been considered by Tranter [10] and Collins [11].

It has been shown $[12,13]$ that all Wiener-Hopf problems can essentially be reduced to the successive solution of two simpler integral equations but there exist certain operators which possess the above factorisation property but which do not seem to be connected with WienerHopf theory. One example of an operator of this type occurs in diffraction theory [14], where the necessary factorisation is derived from Sonine's finite integral formula.

In the present paper the consequences of the factorisation of operators connected with dual series and integral equations into the product of simpler operators is examined from a purely formal point of view. The advantage of a purely formal attack is that methods of solution using particular integral representations and so on for the solution arise naturally from the formalism and require no prior knowledge of results from special function theory.

The formal approach is applied to the two particular examples of dual series equations involving trigonometric functions and of dual integral equations of the type occurring in diffraction theory. From the latter equations it has been found possible to deduce, in a logical fashion, the appropriate integral representation for disk diffraction problems.

2. General Theory. Dual series and dual integral equations may be written in a symbolic fashion as

$$
\begin{array}{ll}
L_{1}(x, \lambda) A(\lambda)=\phi_{1}(x) & (\alpha<x<\beta), \\
L_{2}(x, \lambda) A(\lambda)=\phi_{2}(x) & (\beta<x<\gamma) .
\end{array}
$$


$L_{l}(i=1,2)$ denote linear operators which, in the case of dual integral equations, are defined by relationships of the form

$$
L_{i}(x, \lambda) A(\lambda)=\int K_{i}(x, \lambda) A(\lambda) d \lambda
$$

where the kernels $K_{i}$ are known. For dual series equations the integral over $\lambda$ in equation (3) has to be replaced by a sum over a discrete variable $\mu_{n}$. The operators $L_{i}$ are such that either one or both have unique inverses if $L_{i}(x, \lambda) A(\lambda)$ is given for $\alpha<x<\gamma$, and the solution of

$$
L_{i}(x, \lambda) f(\lambda)=g(x) \quad(\alpha<x<\gamma)
$$

will be written symbolically as

$$
f(\lambda)=L_{i}^{-1}(\lambda, x) g(x)
$$

We make the further assumption that

$$
L_{1}(x, \lambda) L_{2}^{-1}(\lambda, t) g(t)=M_{1}(x, u) M_{2}(u, v, \gamma) g(v) \quad(\alpha<x<\gamma),
$$

where

$$
\begin{gathered}
M_{1}(x, u) f(u)=\int_{\alpha}^{x} N_{1}(x, u) f(u) d u, \\
M_{2}(x, u, \delta) f(u)=\int_{\delta}^{x} N_{2}(x, u) f(u) d u \quad(\alpha<\delta \leqq \gamma)
\end{gathered}
$$

and $N_{1}, N_{2}$ are known functions.

The form of equation (5) appears to be somewhat specialised but all the known types of dual integral and series equations are such that they yield an identity of this type and it is the existence of this factorisation of the operator $L_{1} L_{2}^{-1}$ which forms the basis of all methods of solving equations (1) and (2). The existence of this type of factorisation in a particular example is not generally obvious, but all dual integral equations which can be reduced to a Wiener-Hopf integral equation are such that such a factorisation exists (cf. $[12,13]$ ). There exist, however, certain integral operators involving Bessel functions for which the above type of factorisation exists $[14,15]$ but its existence seems to be in no way connected with WienerHopf theory.

It will also be assumed that the equations

$$
\begin{aligned}
M_{1}(x, u) f_{1}(u)=g_{1}(x) & (\alpha<x<\delta), \\
M_{2}(x, u, \delta) f_{2}(u)=g_{2}(x) & (\alpha<x<\delta)
\end{aligned}
$$

possess unique explicit solutions which will be denoted symbolically by

$$
\begin{array}{ll}
f_{1}(u)=M_{1}^{-1}(u, x) g_{1}(x) & (\alpha<x<\delta), \\
f_{2}(u)=M_{2}^{-1}(u, x, \delta) g_{2}(x) & (\alpha<x<\delta) .
\end{array}
$$


Replacing $g(t)$ in equation (5) by $L_{2}(t, \lambda) h(\lambda)$ and multiplying both sides of the resulting equation by the operator $M_{1}^{-1}$, we obtain

$$
M_{1}^{-1}(u, x) L_{1}(x, \lambda) h(\lambda)=M_{2}(u, v, \gamma) L_{2}(v, \lambda) h(\lambda) \quad(\alpha<u<\gamma) .
$$

Replacing $g$ in equation (5) by $M_{2}^{-1} f$ and multiplying both sides of the resulting equation by $L_{1}^{-1}$, we obtain

$$
L_{1}^{-1}(\lambda, x) M_{1}(x, u) f(u)=L_{2}^{-1}(\lambda, x) M_{2}^{-1}(x, u, \gamma) f(u)
$$

We now examine the three different methods of approach which have been used to solve equations (1) and (2). The first method is that of reducing the dual integral equations to the successive solution of two simple integral equations. This approach was first used by Copson [9] in considering the electrostatic problem for the circular disk.

A function $h(x)$ is defined in $\alpha<x<\beta$ by the equation

and hence

$$
L_{2}(x, \lambda) A(\lambda)=h(x) \quad(\alpha<x<\beta)
$$

$$
L_{1}(x, \lambda) L_{2}^{-1}(\lambda, t) g(t)=\phi_{1}(x) \quad(\alpha<x<\beta),
$$

where $g=h, \alpha<x<\beta, g=\phi_{2}, \beta<x<\gamma$. It now follows from equations (5) and (11) that

$$
M_{1}(x, u) M_{2}(u, v, \beta) h(v)=\phi_{1}(x)-M_{1}(x, u) \int_{\gamma}^{\beta} N_{2}(u, v) \phi_{2}(v) d v \quad(\alpha<x<\beta) .
$$

Two successive inversions of equation (12) give $h(x)$ for $\alpha<x<\beta$; hence $L_{2}(x, \lambda) A(\lambda)$ is known for $\alpha<x<\gamma$ and hence $A(\lambda)$ may be found by a further inversion. The approach seems somewhat cumbersome and is not the best method of finding $A$; in physical problems, however, the quantity $h$ is generally more useful than $A$ and the third inversion to find $A$ can generally be avoided.

The second method of solution is the " multiplying-factor" method which seems to have been first introduced by Noble [12] in connection with certain Wiener-Hopf problems. The basis of this approach is to find two operators $M_{1}^{*}, M_{2}^{*}$ such that the products $M_{1}^{*} L_{1}, M_{2}^{*} L_{2}$ are both equal to the same linear operator $L_{3}$. It follows immediately from equation (8) that $M_{1}^{*}=M_{1}^{-1}, M_{2}^{*}=M_{2}(x, u, \gamma)$ and hence that

$$
M_{2}(x, t, \gamma) L_{2}(t, \lambda) A(\lambda)= \begin{cases}M_{1}^{-1}(x, t) \phi_{1}(t) & (\alpha<x<\beta), \\ M_{2}(x, t, \gamma) \phi_{2}(t) & (\beta<x<\gamma) .\end{cases}
$$

The determination of $A(\lambda)$ from equation (13) would appear to be just as laborious as the first integral equation method but in most examples the product $M_{2} L_{2}$ (i.e. $L_{3}$ ) can be transformed into a simple operator whose inverse can be obtained explicitly. Thus, in general, only two explicit inversions are necessary to give $A(\lambda)$.

The third approach is that of assuming an integral representation of some unknown quantity; there are two slightly different variations of this approach. The first method is that adopted by Tranter [16] who uses an integral representation for the function $h(x)$ defined in equation (10). The second method is that adopted by Gordon [3], Sneddon [5] and Copson 
[4], who assume an integral representation for $A(\lambda)$. Both approaches involve an unknown function which can be shown to satisfy a simple integral equation.

It follows immediately from equation (12) that the appropriate representation for $h(x)$ is $M_{2}^{-1}(x, t, \beta) g(t)$ and $g(t)$ will be determined by

$$
M_{1}(x, t) g(t)=\phi_{1}(x)-\int_{\gamma}^{\beta} N_{2}(x, v) \phi_{2}(v) d v \quad(\alpha<x<\beta) .
$$

From equation (9) it follows that the appropriate representation for $A(\lambda)$ is given by

$$
A(\lambda)=L_{1}^{-1}(\lambda, x) M_{1}(x, u) f(u)=L_{2}^{-1}(\lambda, x) M_{2}^{-1}(x, u, \gamma) f(u)
$$

and hence, from equations (1) and (2),

$$
\begin{aligned}
M_{1}(x, u) f(u) & =\phi_{1}(x) \quad(\alpha<x<\beta), \\
f(u) & =M_{2}(u, x, \gamma) \phi_{2}(x) \quad(\beta<u<\gamma) .
\end{aligned}
$$

In certain cases involving dual series equations the derivation of equation (14) by using an appropriate integral representation can be very involved and require a considerable amount of manipulation involving special functions. This type of situation occurs in trigonometrical dual series considered by Tranter [10]. The operators $M_{1}, M_{2}$ and hence equation (12) can be derived in a very elementary fashion, but an approach based on an integral representation method yields some complicated analysis involving the summation of a series of products of Jacobi Polynomials. For dual integral equations, however, the integral representation approach seems to be more direct $[4,5]$.

The above analysis is purely formal and in particular cases one would have to verify the validity of all the operations. In certain cases the integral (or series) defining $L_{1} L_{2}^{-1}$ may not exist, but in cases where this occurs it has been found that $L_{2} L_{1}^{-1}$ exists and obvious modifications of the above analysis indicate the steps to be taken.

3. Applications. We shall consider briefly two applications of the above formal analysis. The first problem considered is one first solved by Tranter [10] and is the determination of the solution of

$$
\begin{aligned}
& \sum_{n=1}^{\infty} \frac{A_{n}}{n} \sin n x=f(x) \quad(0<x<c), \\
& \sum_{n=1}^{\infty} A_{n} \sin n x=0 \quad(c<x<\pi) .
\end{aligned}
$$

In the notation of the previous section the left hand side of equation (17) is defined to be equal to $h(x)(0<x<c)$ and hence

$$
\int_{0}^{c} h(t) \log \left|\frac{\sin \frac{1}{2}(x+t)}{\sin \frac{1}{2}(x-t)}\right| d t=\pi f(x) \quad(0<x<c) .
$$


Elementary integration gives

$$
\log \left|\frac{x+y}{x-y}\right|=2 \int_{0}^{\min (x, y)} \frac{t d t}{\left(x^{2}-t^{2}\right)^{\frac{1}{t}}\left(y^{2}-t^{2}\right)^{\frac{1}{2}}},
$$

and hence, from equations (17), (18),

where

$$
\cos \frac{1}{2} x \int_{0}^{x} \frac{\tan \frac{1}{2} t S(t) d t}{(\cos t-\cos x)^{\frac{t}{t}}}=\frac{1}{2} \pi f(x) \quad(0<x<c),
$$

$$
S(t)=\int_{t}^{c} \frac{h(x) \cos \frac{1}{2} x d x}{(\cos t-\cos x)^{\frac{1}{2}}} .
$$

Equations (20) and (21) are equivalent to equation (5), the operator $M_{1}$ being defined in equation (20) and $M_{2}$ in equation (21). Inversion of equation (21) gives

$$
h(x) \cos \frac{1}{2} x=-\frac{1}{\pi} \frac{d}{d x} \int_{x}^{c} \frac{S(t) \sin t d t}{(\cos x-\cos t)^{\frac{1}{2}}},
$$

thus defining $M_{2}^{-1}(x, t, c)$. Equation (22) is essentially the starting point of Tranter's solution and equation (20) is deduced from it after considerable manipulation.

As a second example we shall consider dual integral equations of a type which occurs in diffraction theory. The equations to be solved are:

$$
\begin{gathered}
\int_{k}^{\infty} A(\lambda) J_{0}(\lambda x) d \lambda=\phi(x) \quad(0<x<1), \\
\int_{0}^{\infty} A(\lambda)\left(\lambda^{2}-k^{2}\right)^{\frac{1}{2}} J_{0}(\lambda x) d \lambda=0 \quad(x>1) .
\end{gathered}
$$

In the notation of $\$ 2$ we have that

$$
L_{1} L_{2}^{-1} f=\int_{k}^{\infty} J_{0}(\lambda x) \int_{0}^{\infty} \frac{\lambda}{\left(\lambda^{2}-k^{2}\right)^{\frac{1}{2}}} t J_{0}(\lambda t) f(t) d t d \lambda
$$

and it has been shown [14] that the right hand side of equation (23) may be factorised to give

$$
L_{1} L_{2}^{-1} f=\frac{2}{\pi} \int_{0}^{x} \frac{\cos k\left(x^{2}-t^{2}\right)^{\frac{1}{2}}}{\left(x^{2}-t^{2}\right)^{\frac{1}{2}}} \int_{t}^{\infty} \frac{\cos k\left(w^{2}-t^{2}\right)^{\frac{1}{2}}}{\left(w^{2}-t^{2}\right)^{\frac{1}{2}}} w f(w) d w d t,
$$

which is of the same form as equation (5). We shall now show that the formal analysis of $\$ 2$ enables an appropriate integral representation for $A$ to be deduced in a logical fashion.

The solution of

$$
\int_{x}^{1} \frac{\cos k\left(u^{2}-x^{2}\right)^{\frac{1}{2}}}{\left(u^{2}-x^{2}\right)^{\frac{1}{2}}} u f(u) d u=g(x)
$$


is known to be [17]

$$
f(x)=-\frac{2}{\pi x} \frac{d}{d x} \int_{x}^{1} \frac{\cosh k\left(u^{2}-x^{2}\right)^{\frac{1}{2}}}{\left(u^{2}-x^{2}\right)^{\frac{1}{2}}} u g(u) d u .
$$

Thus, in the notation of the previous section, the operator $M_{2}^{-1}(x, u, 1)$ is defined by the right hand side of equation (25). It thus follows from equations (15) and (25) that a suitable choice for $A(\lambda)$ is given by

$$
A(\lambda)=\frac{\lambda}{\left(\lambda^{2}-k^{2}\right)^{\frac{1}{2}}} \int_{0}^{1} J_{0}(\lambda x)\left[\frac{d}{d x} \int_{x}^{1} \frac{\cosh k\left(u^{2}-x^{2}\right)^{\frac{1}{2}}}{\left(u^{2}-x^{2}\right)^{\frac{1}{2}}} u g(u) d u\right] d x,
$$

where $g(u)=0(u>1)$. Integration by parts in (26) and slight manipulation shows that

$$
A(\lambda)=-\frac{\lambda}{\left(\lambda^{2}-k^{2}\right)^{\frac{1}{2}}} \int_{0}^{1} g(t) \cos t\left(\lambda^{2}-k^{2}\right)^{\frac{1}{2}} d t .
$$

For $k=0$ the representation of equation (27) reduces to that used by Sneddon [5], and starting from equation (25) one can obtain an elementary solution of the above equations in exactly the same manner as Sneddon [5] obtained such a solution for the case $k=0$.

In diffraction problems the dual integral equations arise from imposing certain boundary conditions at $z=0$ on the function

$$
V=\int_{0}^{\infty} e^{-1=1\left(\lambda^{2}-k^{2}\right)^{1 / 2}} A(\lambda) J_{0}(\lambda \rho) d \lambda .
$$

If the above form for $A(\lambda)$ is now substituted in the expression for $V$, it follows after slight manipulation that

$$
V=-\int_{0}^{1} g(t)\left[\frac{\exp i k\left\{(z+i t)^{2}+\rho^{2}\right\}^{\frac{1}{2}}}{\left\{(z+i t)^{2}+\rho^{2}\right\}^{\frac{1}{2}}}+\frac{\exp i k\left\{(z-i t)^{2}+\rho^{2}\right\}^{\frac{1}{2}}}{\left\{(z-i t)^{2}+\rho^{2}\right\}^{\frac{1}{2}}}\right] d t .
$$

The above form of $V$ is essentially the starting point of the work of Bazer and Brown, and Collins, in treating various diffraction problems.

From Sonine's second finite integral formula and the Hankel inversion theorem, a generalisation of the factorisation of equation (24) may be obtained [15]. From this general factorisation, dual integral equations of the above type with Bessel functions of non-zero order may be solved [15]. Equations of this type also appear to have been considered independently by Peters in some unpublished work (cf. $[8,18]$ ).

\section{REFERENCES}

1. E. C. Titchmarsh, Theory of Fourier integrals (Oxford, 1937), p. 334.

2. I. W. Busbridge, Dual integral equations, Proc. London Math. Soc. 44 (1938), 115-129.

3. A. N. Gordon, Dual integral equations, J. London Math. Soc. 29 (1954), 360-363.

4. E. T. Copson, On certain dual integral equations, Proc. Glasgow Math. Assoc. 5 (1961), 21-24.

5. I. N. Sneddon, The elementary solution of dual integral equations, Proc. Glasgow Math. Assoc. 4 (1960), 108-110.

6. B. Noble, Certain dual integral equations, J. Math. Phys. 37 (1955), 128-136. 
7. W. E. Williams, Solution of certain dual integral equations, Proc. Edinburgh Math. Soc. 12 (1961), 213-216.

8. A. Erdélyi and I. N. Sneddon, Fractional integration and dual integral equations, Canad. J. Math. 14 (1962), 685-693. $14-19$.

9. E. T. Copson, On the problem of the electrified disc, Proc. Edinburgh Math. Soc. 8 (1947),

10. C. J. Tranter, Dual trigonometrical series, Proc. Glasgow Math. Assoc. 4 (1960), 49-57.

11. W. D. Collins, On some dual series equations and their applications to electrostatic problems for spheroidal caps, Proc. Cambridge Philos. Soc. 57 (1961), 367-384.

12. B. Noble, Wiener-Hopf methods (Oxford, 1958), p. 230. 120.

13. W. E. Williams, A class of mixed boundary value problems, J. Math. Mech. 11 (1962), 109-

14. W. E. Williams, Diffraction by a disk, Proc, Roy. Soc. Ser. A 267 (1962), 77-87.

15. W. E. Williams, A class of integral equations, Proc. Cambridge Philos. Soc. 59 (1963), 589-597.

16. C. J. Tranter, On some dual integral equations, Quart. J. Math. Oxford Ser. (2) 2 (1951), 60-66.

17. D. S. Jones, A new method for calculating scattering with particular reference to the circular disk, Comm. Pure Appl. Math. 9 (1956), 713-746.

18. I. N. Sneddon, Fractional integration and dual integral equations (North Carolina State College, June 1962).

THB UNIVERSITY

LIVERPOOL 